Audiology

Neurotology
Audiol Neurotol 2009;14:308-314

DOI: $10.1159 / 000212110$
Received: August 22, 2008

Accepted after revision: November 19, 2008

Published online: April 15, 2009

\title{
Mastoid Cavity Dimensions and Shape: Method of Measurement and Virtual Fitting of Implantable Devices
}

\author{
Ophir Handzel $^{\mathrm{a}-\mathrm{c}}$ Haobing Wang ${ }^{\mathrm{b}}$ Jason Fiering ${ }^{\mathrm{e}}$ Jeffrey T. Borenstein ${ }^{\mathrm{e}}$ \\ Mark J. Mescher ${ }^{e}$ Erin E. Leary Swan ${ }^{e, f}$ Brian A. Murphy ${ }^{b} Z_{\text {Zhiqiang Chen }}{ }^{a-c}$ \\ Marcello Peppi ${ }^{a-c}$ William F. Sewell ${ }^{a-d}$ Sharon G. Kujawa ${ }^{a, c}$ \\ Michael J. McKennaa, c \\ ${ }^{a}$ Department of Otolaryngology and ${ }^{b}$ Eaton Peabody Laboratory, Massachusetts Eye and Ear Infirmary, \\ 'Department of Otology and Laryngology and d Program in Neuroscience, Harvard Medical School, Boston, Mass., \\ and ${ }^{e}$ Draper Laboratory, and ${ }^{\mathrm{f}}$ Department of Mechanical Engineering, Massachusetts Institute of Technology, \\ Cambridge, Mass., USA
}

\section{Key Words}

Mastoid dimension - Mastoid size measurement method •

Implantable device

\begin{abstract}
Temporal bone implants can be used to electrically stimulate the auditory nerve, to amplify sound, to deliver drugs to the inner ear and potentially for other future applications. The implants require storage space and access to the middle or inner ears. The most acceptable space is the cavity created by a canal wall up mastoidectomy. Detailed knowledge of the available space for implantation and pathways to access the middle and inner ears is necessary for the design of implants and successful implantation. Based on temporal bone CT scans a method for three-dimensional reconstruction of a virtual canal wall up mastoidectomy space is described. Using Amira ${ }^{\circledR}$ software the area to be removed during such surgery is marked on axial CT slices, and a three-dimensional model of that space is created. The average volume of 31 reconstructed models is $12.6 \mathrm{~cm}^{3}$ with standard deviation of 3.69 $\mathrm{cm}^{3}$, ranging from 7.97 to $23.25 \mathrm{~cm}^{3}$. Critical distances were measured directly from the model and their averages were
\end{abstract}

calculated: height $3.69 \mathrm{~cm}$, depth $2.43 \mathrm{~cm}$, length above the external auditory canal (EAC) $4.45 \mathrm{~cm}$ and length posterior to EAC $3.16 \mathrm{~cm}$. These linear measurements did not correlate well with volume measurements. The shape of the models was variable to a significant extent making the prediction of successful implantation for a given design based on linear and volumetric measurement unreliable. Hence, to assure successful implantation, preoperative assessment should include a virtual fitting of an implant into the intended storage space. The above-mentioned three-dimensional models were exported from Amira to a Solidworks application where virtual fitting was performed. Our results are compared to other temporal bone implant virtual fitting studies. Virtual fitting has been suggested for other human applications.

Copyright $\odot 2009$ S. Karger AG, Basel

\section{Introduction}

Implantable devices can be used to treat a variety of ear diseases. Cochlear implants are the most common implanted devices. Other devices amplify sound by vibrating the ossicles [Bakous and Duke, 2006], the round window

\section{KARGER}

๑ 2009 S. Karger AG, Basel

Fax +41 613061234 E-Mail karger@karger.ch www.karger.com www.karger.com/aud
Michael J. McKenna

Department of Otolaryngology

Massachusetts Eye and Ear Infirmary

243 Charles Street, Boston, MA 02115 (USA)

Tel. +1 617573 4403, Fax +1 617573 3939, E-Mail michael_mckenna@meei.harvad.edu 
membrane [Colletti et al., 2006] or by direct coupling to inner ear fluids [Bernhard et al., 2006]. Finally, implantable inner ear drug delivery systems through the round window have been described [Lehner et al., 1997; Chen et al., 2005; Plontke et al., 2006], and it is likely that other designs will follow for this and other clinical applications [Swan et al., 2008].

When storage and access space are required for implantable devices, a cavity is often created by a canal wallup mastoidectomy, a function-preserving procedure. The middle ear or a cochleostomy site can be reached from the mastoid space through the facial recess, the standard approach for cochlear implants. Although space and access are rarely a limiting factor for cochlear implants they can be an important consideration for the design and applicability of many other ear implants.

As the field of implantable devices evolves normative data regarding size and shape restrictions for future designs are required. Multiple studies have provided data regarding the dimension of the air cell space of the mastoid, but this space includes volume not amenable to implantation and does not include spaces suitable for implantation (i.e. the mastoid cortex). To date, one set of measurements of the size of the implantable space has been published [Maassen et al., 1997]. This space varies between individuals in volume, linear dimensions (height, depth etc.) and shape. As a result, evaluation of individual candidates for implantation must also include an assessment of the space available in the temporal bone to be implanted [Dammann et al., 2001; Maassen et al., 2004]. Hence, a reliable, clinically applicable, and cost- and time-efficient method for evaluating candidates for implantation is required. The goal of this study is to provide additional data about the dimension of the space available for implantation and to refine further a method for preoperative evaluation of future candidates for temporal bone-implantable devices.

\section{Material and Methods}

Measurements were made based on axial high resolution CT scans of the temporal bones obtained from patients at the Massachusetts Eye and Ear Infirmary. Scans of 31 temporal bones of 31 patients were retrospectively allocated for the study. The scans were requested for non-study-related clinical purposes, most commonly for inquiries regarding the contralateral ear and to rule out superior semicircular dehiscence. Patients with pathologies involving the temporal bone by history or imaging were excluded. Chronic otitis media was a common exclusion criterion. No patient identifiers were collected. Scans were obtained by a Siemens Somaton Sensation (New York, N.Y., USA) spiral CT scanner in 40-slice mode using the following parameters: 320 MAS, $150 \mathrm{~mm}$ FOV, $120 \mathrm{kV}$ and $0.6 \mathrm{~mm}$ collimator. Reconstruction was done by a $0.6 \times 0.2 \mathrm{~mm}$ high resolution bone window for each temporal bone separately using $200 \mathrm{~mm}$ FOV.

The axial slices of the CT scans of the temporal bones were used as a basis for reconstructing a three-dimensional (3D) model with Amira ${ }^{\circledR}$ (Mercury computer systems, Chelmsford, Mass., USA). Amira ${ }^{\circledR}$ is well suited for the creation of $3 \mathrm{D}$ models from DICOM (Digital Imaging and Communications in Medicine) files (the output of CT scans). Reconstruction of a 3D model involved manual selection of areas in the axial slices and automated conversion of the selected areas to a 3D model. The first author (a neurotology fellow) manually marked the area that would have been removed during a canal wall-up mastoidectomy and the facial recess approach to the middle ear on every other axial section of the CT scan. The markings on the rest of the axial sections were generated by interpolation, which is a marking tool in Amira ${ }^{\circledR}$ that can generate new outlines following closely the existing irregular contours of a cavity. The accuracy of the marking by interpolation was confirmed for each section by visual inspection of the intermediate section confirming accurate marking of the anatomical and surgical boundaries of the space. Using Amira ${ }^{\circledR}$ these axial slice markings were reconstructed to a $3 \mathrm{D}$ model of the surgical defect (fig. 1a-c). The reconstructed model is a surface model made of small triangles. The model was smoothed by reducing the number of triangles composing its surface and by removing redundant vertices. Smoothing the surface makes the model better reflect the true space by eliminating small irregularities inherent to the manual marking process. These irregularities stem from the differences in the marking in adjacent stacked sections. To assure that smoothing was not exaggerated, for a sample of models, measurements of the unsmoothed model were compared to the smoothed model. Linear and volumetric measurements varied between the models by less than $1 \%$. The output of the $3 \mathrm{D}$ reconstruction is a mesh format representation of an envelope containing the desired space and is encoded as a STL file (a common mesh format).

For purposes of optimizing a prototype device to fit in the space, the mesh model was subsequently converted to a boundary representation solid model (3D objects defined by geometric boundaries and faces) encoded as an IGES file. The latter solid model was imported to a Solidworks (Solidworks Corp., Concord, Mass., USA) application, which was used for virtual fitting of the device into the cavity. Virtual fitting involved an attempt to completely place a model of an implant into the $3 \mathrm{D}$ virtual cavity. During the conversion to the IGES file further smoothing of the model was required to reduce the data file to a manageable size. The final model was reevaluated to confirm that it accurately reflects the original cavity. Alternately, the solid model of the device could be exported in STL format and the virtual fitting could be performed in Amira ${ }^{\circledR}$.

Amira ${ }^{\circledR}$ allows for linear measurements to be made directly from the 3D model. Compared to measurements from CT slices these are easier to obtain and are more likely to accurately represent the true dimensions of the space. Linear measurements of the cavity included: height, depth (distance from mastoid outer cortex to medial edge of the model), anterior-posterior distance above the external auditory canal (EAC) and posterior to the EAC (fig. 1b, c). The facial recess space was not included in the volumetric measurements of the surgical cavity. The distance from the facial recess to the endosteal side of the cochlea at the area of the round window (inward from the operculum of the round window 

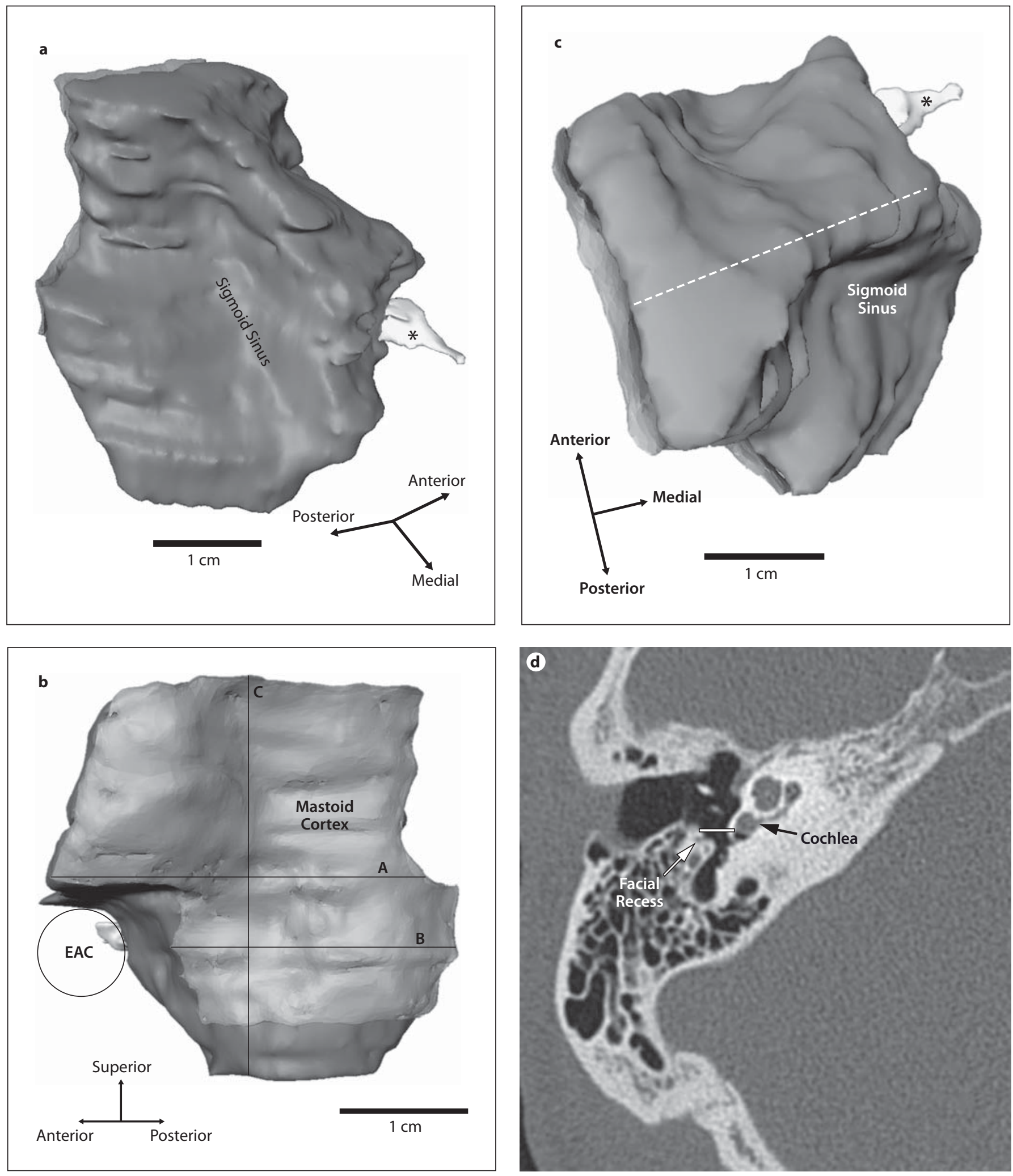
niche) was measured as well (fig. 1d). For implantable inner ear drug delivery systems this is a distance that needs to be gapped between the device (in the mastoid) and the beginning of the perilymphatic space. Reconstruction from axial CT scans to a $3 \mathrm{D}$ model took $45 \mathrm{~min}$ on an average, file formatting and virtual fitting required additional time.

\section{Results}

The volumetric and linear measurements for 31 reconstructed 3D models of temporal bones are summarized in table 1 and in figure 2. The volume of the space created by a virtual canal wall-up mastoidectomy ranged from 7.97 to $23.25 \mathrm{~cm}^{3}$ averaging $12.63 \mathrm{~cm}^{3}$ with standard deviation of $3.69 \mathrm{~cm}^{3}$. Ninety percent of the $3 \mathrm{D}$ models had volumes of $8.5 \mathrm{~cm}^{3}$ or larger. Critical distances were measured directly from each model. The height (superior-inferior dimension, fig. 1b) averaged $3.69 \mathrm{~cm}$ with a standard deviation of $0.75 \mathrm{~cm}$. The depth (medial-lateral dimension, fig. 1c) averaged $2.43 \mathrm{~cm}$ with standard deviation of 0.28 $\mathrm{cm}$. The length above the EAC (anterior-posterior dimension, fig. 1b) averaged $4.45 \mathrm{~cm}$ with standard deviation of $0.82 \mathrm{~cm}$. The length posterior to the EAC averaged $3.16 \mathrm{~cm}$ with standard deviation of $0.41 \mathrm{~cm}$. The distance from the facial recess to the cochlea (fig. 1d) averaged $0.52 \mathrm{~cm}$ with standard deviation of $0.07 \mathrm{~cm}$ (table 1).

Linear measurements did not correlate well with the volume of the cavity. Correlation coefficients for the linear measurements and volume were (fig. 3): height 0.53, depth 0.63 , length above EAC 0.76, and length behind EAC 0.61. Age correlated poorly with cavity volume (correlation coefficient 0.16 ).

Fig. 1. Images of the reconstructed 3D model of the space created during a canal wall-up mastoidectomy that can be used for storage of an implantable device, and the linear measurements taken. a View of posterior-medial plane of the cavity. The impression of the sigmoid sinus is marked. The facial recess and access to the cochlea is seen bulging from the medial aspect of the model (marked by an asterisk). b Lateral view, the opening from the cortex to the cavity is marked in a lighter color. This is the opening available for insertion of an implantable device. The cortex over the tip of the mastoid was not removed to reduce the likelihood of medial auricle depression and deformity. Linear measurements are marked: $\mathrm{A}=$ Length above the EAC; $\mathrm{B}=$ length posterior to EAC; $\mathrm{C}=$ height. $\mathrm{c}$ Superior view. The dashed line depicts the measurement of the width of the model, which is actually embedded inside the model. The impression of the sigmoid sinus is marked. d Axial temporal bone CT scan demonstrating the distance between the facial recess and the cochlea. This distance must be gapped in order to reach the inner ear from the mastoid space.

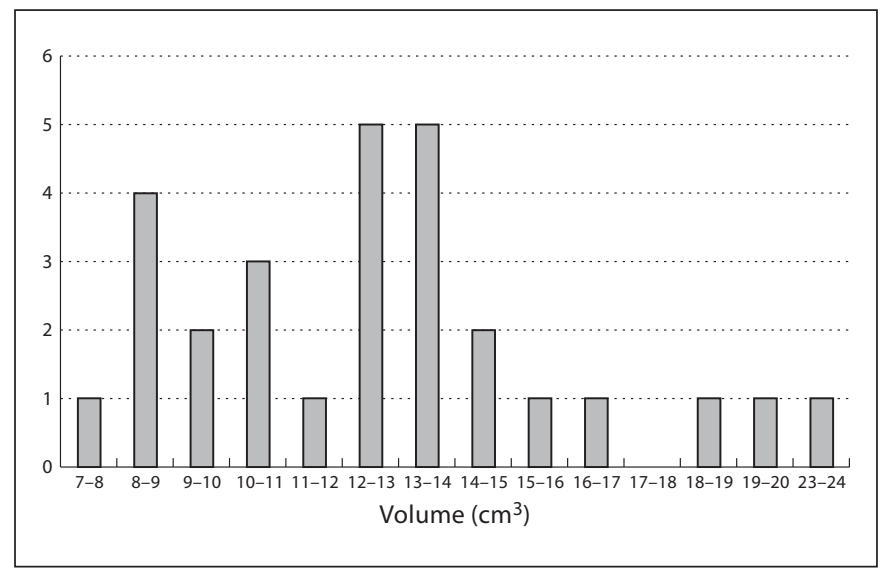

Fig. 2. Histogram depicting distribution of volume sizes in cubic centimeters of the virtually created mastoid cavities. The y axis depicts the number of subjects in each size group.

\section{Discussion}

Temporal bone implants can be used to electrically stimulate the auditory nerve, to amplify sound, to deliver drugs to the inner ear and potentially for other future applications. The development of implantable devices for inner ear drug delivery is on the increase [Lehner et al., 1997; Chen et al., 2005; Plontke et al., 2006; Swan et al., 2008]. When compared to systemic and intratympanic routes, drug delivery directly to the inner ear has many potential advantages: higher concentration of drug in the target organ, control of the amount, rate and timing of drug delivery, the option for long-term continuous treatment and reduced systemic side effects. It is likely that in the future more devices will be made available and indications for implantation broadened.

The implants require storage space and access to the middle or inner ears. The most acceptable storage space is the one created by a canal wall-up mastoidectomy. This procedure does not interfere with the normal functions of the ear. From the cavity, the cochlea and middle ear can be approached through the facial recess. The design of implantable devices must take into consideration the restrictions on shape and dimension dictated by the space available in the mastoid cavity. The actual available space for storage is larger than the one created by mastoidectomy, as some components can be located in a more posterior or superior surgically created cortical depression, similar to cochlear implants. A battery seems to be a potentially suitable component for this location. However, for some components and applications, the mastoid cav- 
Table 1. Data from measurements of virtually created canal wall-up mastoidectomy cavities based on $31 \mathrm{CT}$ scans of human temporal bones

\begin{tabular}{|c|c|c|c|c|c|c|c|c|}
\hline No. & Side & $\begin{array}{l}\text { Age } \\
\text { years }\end{array}$ & $\begin{array}{l}\text { Volume } \\
\mathrm{cm}^{3}\end{array}$ & $\begin{array}{l}\text { Height } \\
\mathrm{cm}\end{array}$ & $\begin{array}{l}\text { Depth } \\
\mathrm{cm}\end{array}$ & $\begin{array}{l}\text { Length } \\
\text { above } \\
\text { EAC, cm }\end{array}$ & $\begin{array}{l}\text { Length } \\
\text { behind } \\
\text { EAC, cm }\end{array}$ & $\begin{array}{l}\text { Distance from } \\
\text { FR to cochlea } \\
\mathrm{cm}\end{array}$ \\
\hline 1 & right & 17 & 7.97 & 3.37 & 2.09 & 3.72 & 2.45 & 0.5 \\
\hline 2 & left & 21 & 8.34 & 3.17 & 2.11 & 3.71 & 3.02 & 0.55 \\
\hline 3 & right & 30 & 8.4 & 3.08 & 2.03 & 3.81 & 3.09 & 0.52 \\
\hline 4 & right & 30 & 8.66 & 2.93 & 2.19 & 4.17 & 2.92 & 0.42 \\
\hline 5 & left & 49 & 8.7 & 3.13 & 2.07 & 2.97 & 3.38 & 0.45 \\
\hline 6 & left & 24 & 8.9 & 3.31 & 2.34 & 3.22 & 2.73 & 0.66 \\
\hline 7 & left & 78 & 9.05 & 2.91 & 2.58 & 4.21 & 2.97 & 0.49 \\
\hline 8 & left & 23 & 9.11 & 3.01 & 2.64 & 3.57 & 2.69 & 0.45 \\
\hline 9 & right & 35 & 9.84 & 2.86 & 2.49 & 4.38 & 3.47 & 0.5 \\
\hline 10 & right & 27 & 10.4 & 4.64 & 2.37 & 3.68 & 2.57 & 0.41 \\
\hline 11 & right & 44 & 10.5 & 3.78 & 2.13 & 4.07 & 2.42 & 0.5 \\
\hline 12 & right & 37 & 10.8 & 3.3 & 2.26 & 5.39 & 3.28 & 0.43 \\
\hline 13 & right & 64 & 11.99 & 3.57 & 2.49 & 3.61 & 3.03 & 0.48 \\
\hline 14 & left & 34 & 12.1 & 3.74 & 2.41 & 4.68 & 3.41 & 0.41 \\
\hline 15 & right & 40 & 12.2 & 3.54 & 2.21 & 3.85 & 3.34 & 0.57 \\
\hline 16 & left & 46 & 12.6 & 3.97 & 2.19 & 3.89 & 2.78 & 0.47 \\
\hline 17 & right & 52 & 12.8 & 3.56 & 2.26 & 4.59 & 3.34 & 0.46 \\
\hline 18 & right & 54 & 12.84 & 4.9 & 2.39 & 4.41 & 2.95 & 0.58 \\
\hline 19 & right & 31 & 13.02 & 3.5 & 2.25 & 4.55 & 3.4 & 0.61 \\
\hline 20 & right & 29 & 13.06 & 3.92 & 2.37 & 4.52 & 3.33 & 0.46 \\
\hline 21 & left & 30 & 13.19 & 3.42 & 2.29 & 4.32 & 2.95 & 0.49 \\
\hline 22 & right & 16 & 13.44 & 4.37 & 2.45 & 4.83 & 3.05 & 0.63 \\
\hline 23 & right & 76 & 13.5 & 4.44 & 2.74 & 3.77 & 3.35 & 0.66 \\
\hline 24 & left & 29 & 14.02 & 4.16 & 2.36 & 4.97 & 3.12 & 0.54 \\
\hline 25 & right & 20 & 14.27 & 4.19 & 2.87 & 5.31 & 2.97 & 0.53 \\
\hline 26 & left & 68 & 15.89 & 3.43 & 2.66 & 5.63 & 3.28 & 0.48 \\
\hline 27 & right & 15 & 16.31 & 3.64 & 3.08 & 6.22 & 4.33 & 0.65 \\
\hline 28 & left & 65 & 18.26 & 3.79 & 2.51 & 5.51 & 3.79 & 0.47 \\
\hline 29 & right & 81 & 18.6 & 4.97 & 2.58 & 4.87 & 3.21 & 0.53 \\
\hline 30 & right & 24 & 19.46 & 3.91 & 3.14 & 5.91 & 3.34 & 0.52 \\
\hline \multirow[t]{3}{*}{31} & right & 28 & 23.25 & 3.97 & 2.63 & 5.55 & 3.92 & 0.62 \\
\hline & Average & 39.26 & 12.63 & 3.69 & 2.43 & 4.45 & 3.16 & 0.52 \\
\hline & $\mathrm{SD}$ & 19.29 & 3.69 & 0.57 & 0.28 & 0.82 & 0.41 & 0.07 \\
\hline
\end{tabular}

ity is a mandatory storage and access space. Consequently, detailed knowledge of the shape and dimension of the space available for implantation is essential.

Measuring the mastoid cavity can be achieved by a number of methods. The ideal method of measurement would represent the shape and dimension of the space accurately, will be applicable for the evaluation of future candidates for implantation and will be efficient in its requirements for time and equipment. A cavity created in a cadaver temporal bone can be measured by imaging, by volume of fluid or by creating a model by impression of the cavity and measuring the dimensions of the physical model. Measurements can also be made based on CT scans of temporal bones of patients who have undergone canal wall-up mastoidectomies. Based on CT scans of temporal bones, a virtually created cavity can be made and measured. Each of these methods has disadvantages. Methods based on cadaver bones are not suitable for evaluating candidates for implantation. Imaging of patients who have undergone mastoidectomy may represent the cavity inaccurately due to imperfections in surgical technique and postoperative changes in the bone. Construction of a virtual 3D model based on CT scans of the temporal bones, as reported here, has the decisive advantage of being suitable for evaluation of future candidates for device implantation. As part of the preoperative assessment a trial of virtual fitting 


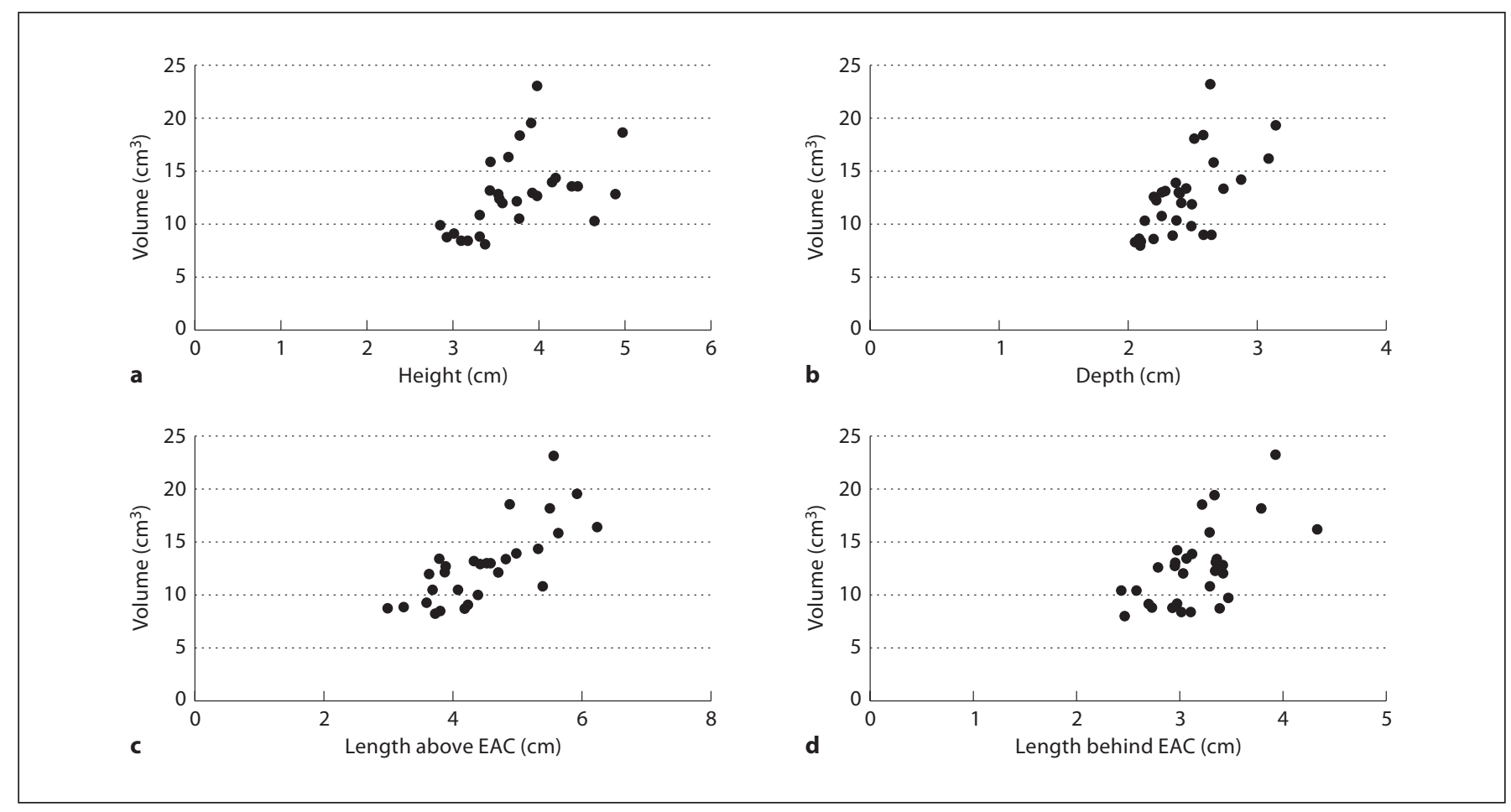

Fig. 3. Scatter plots and correlation coefficients between linear measurement of size and volumes. Correlation coefficients between linear measurements and volumes: height 0.53 (a), depth 0.63 (b), length above EAC 0.76 (c), length behind EAC 0.61 (d). Correlations are not sufficient to use any linear measurement as a predictor for the volume.

of the device to the candidate's $3 \mathrm{D}$ reconstructed cavity can eliminate the possibility of aborting a surgery due to shape and/or dimension inadequacy. The choice of software for $3 \mathrm{D}$ reconstruction was based on its suitability for input of DICOM (CT scan output) files, compatibility of interface with engineering specific software and ease of use.

Based on analysis of 31 temporal bone CT scans, the average volume of a canal wall-up mastoid space is 12.63 $\mathrm{cm}^{3}$ with standard deviation of $3.69 \mathrm{~cm}^{3}$, ranging from 7.97 to $23.25 \mathrm{~cm}^{3}$. These dimensions exclude subjects with temporal bone pathology and chronic otitis media by history or imaging, as the disease process can interfere with normal mastoid development and aeration, resulting in smaller cavities. Maassen et al. studied the size of the mastoid cavity of 50 cadaver temporal bones [Maassen et al., 1997]. Based on CT scans, the average size of a virtual cavity was $8.56 \mathrm{~cm}^{3}$ with standard deviation of $3.59 \mathrm{~cm}^{3}$. The same group also measured the size of the cavity after performing a canal wall-up mastoidectomy on the same 50 temporal bones. The average volume of reconstructed actual cavities created in these bones was $7.81 \mathrm{~cm}^{3}$ and standard deviation of $2.97 \mathrm{~cm}^{3}$. The correla- tion coefficient between the virtually marked and actual cavities was 0.79 . The smaller average cavity size in the study of Maassen et al. [1997] compared with our findings may be the result of our exclusion of patients with a history of chronic otitis media and differences between the exact areas included in the reconstruction.

Linear measurements were not well correlated to volumes and are not reliable predictors for successful implantation (fig. 3). This finding is to be expected given the large individual variance in the shape of the cavity and is in agreement with the findings of Maassen et al. [1997]. The shape of the cavity depends on the extent and pattern of aeration of the mastoid bone and these vary greatly among individuals.

In contrast to linear measurements, volume seems to better prognosticate successful implantation. In a cadaver temporal bone CT study, Maassen et al. [1997] reported a sensitivity of $89.7 \%$ for predicting successful implantation of a device of their design, with a positive predictive value of $100 \%$ (successful implantation was correctly anticipated) and $73.3 \%$ negative predictive value (the probability of correct negative prediction). These values 
may vary according to the specific design of the implant. Although cavity volume is a better predictor of implantation result, it is not fail-proof due to variation in the shape of the cavity. For example, although subjects 10 and 11 have a near-identical volume of the mastoid space, the shapes of their cavities are far from identical, one being higher and shorter than the other (table 1). Hence, there is a need for individual virtual fitting of an implantable device to a virtually created surgical defect. Preoperative $3 \mathrm{D}$ virtual assessment seems to be the most appropriate tool for avoiding unnecessary surgery aborted by undersized or unusually shaped cavities.

Imaging-based $3 \mathrm{D}$ reconstruction and virtual fitting have been used in other medical specialties [Shuxian et al., 2005; Saeed et al., 2008]. Virtual fitting has been validated compared to implant surgery in cadaver temporal bones and in patients [Maassen et al., 2004]. In this prospective study, 15 cadaver temporal bones and 24 candidates for implantation of a device designed by the researchers were evaluated for suitability for implantation by virtual fitting. The results of the virtual fitting were compared to success of implantation during actual surgery. The virtual fitting was accurate in all cases with the exception of two false positives, one in a cadaver and the other in a patient (successful implantation predicted but not achieved during surgery). The patient's false positive was caused by a component not evaluated by the virtual fitting process.

We based our measurements on manual markings on axial sections of temporal bone CT scans. Although the basic borders of the cavity created by a canal wall-up mas- toidectomy are well defined, accuracy of the marking process is operator-dependent. Borders were marked based on a conservative estimate of the bone and air cells to be removed, paying special attention to prevent interference with normal function of the ossicles. Reconstruction of each model took an average of $45 \mathrm{~min}$, comparable to other published data [Dammann et al., 2001]. Virtual fitting of the device in the cavity requires additional time. Although less expeditious than ideal, it is still a reasonable time to spend on studying future candidates.

In conclusion, temporal bone implantable devices for sound amplification, for direct delivery of drugs to the inner ear and for other indications are likely to become more prevalent in the future. The design of such devices requires detailed knowledge of the dimension and shape of the available storage and access spaces. Based on high resolution CT scans, 3D reconstruction of a virtual canal wall-up mastoidectomy cavity provides the necessary shape and size data regarding the available space for such designs. The same process can be used for the evaluation of candidates for implantation by an attempt at virtual fitting of such devices. This study includes data about the size and dimension at the implantable space as well as a suggested method for the evaluation of future candidates for implants.

\section{Acknowledgments}

Supported by NIH/NIDCD grant 5R01DC006848. Ophir Handzel is partially supported by the American Physicians Fellowship program.

\section{References}

Bakous DD, Duke W: Implantable middle ear hearing devices: current state of technology and market challenges. Curr Opin Otolaryngol Head Neck Surg 2006;14:314-318.

-Bernhard H, Stieger C, Perriard Y: New implantable hearing device based on a micro-actuator that is directly coupled to the inner ear fluid. Conf Proc IEEE Eng Med Biol Soc 2006;1:3162-3165.

-Chen Z, Kujawa SG, McKenna MJ, Fiering JO, Mescher MJ, Borenstein JT, Swan EE, Sewell WF: Inner ear drug delivery via a reciprocating perfusion system in the guinea pig. J Control Release 2005;110:1-19.

Colletti V, Soli SD, Carner M, Colletti L: Treatment of mixed hearing losses via implantation of a vibratory transducer on the round window. Int J Audiol 2006;45:600-608.
Dammann F, Bode A, Schwaderer E, Schaich M, Heuschmid M, Maassen MM: Computeraided surgical planning of hearing aids based on CT data in VR environment. Radiographics 2001;21:183-190.

Lehner R, Brugger H, Maassen MM, Zenner HP: A totally implantable drug delivery system for local therapy of the middle and inner ear. Ear Nose Throat J 1997;76:567-570.

- Maassen MM, Lehner R, Ludtke R, Strayler-Batra M, Zenner HP: Preoperative assessment of the implantable middle ear pump system using CT scans and conventional X-rays of the temporal bone. Ear Nose Throat J 1997; 76:457-463.

Maassen MM, Schwaderer E, Heinrich B, Herberhold S, Mauz PS, Dammann F: Comparison of the implantability of electronic hearing devices in a virtual reality planning environment and in human temporal bones. Acta Otolaryngol 2004;124:1039-1045.
PlontkeSK, Zimmermann R, Zenner HP, Löwenheim $\mathrm{H}$ : Technical note on microcatheter implantation for local inner ear drug delivery: surgical technique and safety aspects. Otol Neurotol 2006;27:912-917.

-Saeed D, Ootaki Y, Noecker A, Weber S, Smith WA, Duncan BW, Fukamachi K: The Cleveland Clinic PediPump: virtual fitting studies in children using three-dimensional reconstructions of cardiac computed tomography scans. ASAIO J 2008;54:133-137.

-Shuxian Z, Wanhua Z, Bingheng L: 3D reconstruction of the structure of a residual limb for customising the design of a prosthetic socket. Med Eng Phys 2005;27:67-74.

- Swan EE, Mescher MJ, Sewell WF, Tao SL, Borenstein JT: Inner ear drug delivery for auditory applications. Adv Drug Deliv Rev 2008; 60:1583-1599. 